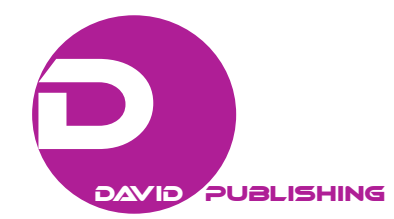

\title{
Explore the Aromatherapy Effect and Performance by Using Questionnaire and Neuroscience Technology
}

\author{
Liu Chia-Ju \\ Open University of Kaohsiung, Kaohsiung, Taiwan \\ Hsieh Chen \\ National Kaohsiung Normal University, Kaohsiung, Taiwan
}

\begin{abstract}
The purpose of this study is to explore the aromatherapy effect and performance by using questionnaire and neuroscience technology. There are different concentration of aromatherapy (this study adapted in lavender) in this study and all participants will perform their attention and anxious after smelling these different concentration of aromatherapy. This study adopted vapor extraction to extract the nature aroma and odor. There are 30 volunteers participated in the study, all of the participants need to write down the questionnaires about attention and anxious, and would smell with carrying the brain wave cap to detect their neuroscience data. The results show that higher concentration of aromatherapy could induce the participants' attention, but lower concentration of aromatherapy could let the participants' feel more relax. Furthermore, no matter the higher or lower concentration of aromatherapy could induce the participants' higher attention and decrease their anxious than control task.
\end{abstract}

Keywords: aromatherapy, anxious, neuroscience

\section{Introduction}

Nowadays, more and more human beings have emotional barriers and could not control their emotion reflections well. A lot of researchers inferred that the possible reasons are that the human beings in the present age have huge anxious and did not contact with nature life right now (Nguyen \& Paton, 2008). Pekrun, Goetz, Titz, and Perry (2002) also mentioned that some kind of carriers which need to pay more attentions or patients to serve customers would cause the workers' high anxious, such as doctors, nurses, teachers, and servers. We may think that if a teacher had bad mood in the class, the students will be induced the bad emotion in the class. As the same, if a doctor or a nurse could not control their emotion, the patients would be induced the bad mood in the medical treatment.

For helping people feel relax, a lot of nature aromatherapy would be extraction to produce as fragrance or body location. People could decrease their anxious by smelling the flavor from nature aromatherapy (Herz, 2009). In Taiwan, most people like the flavor of lavender to be a nature aromatherapy for relaxing (Liu, Yang, Chen, \& Wang, 2017). Liu et al.'s research found that the patients would decrease their anxious and feel more relax after smelling the nature lavender aromatherapy. In the past, a lot of studies supported that the flavor could transmit to human's brain from nose and affect the processing of our brain area, such as prefrontal cortex,

Liu Chia-Ju, president, Open University of Kaohsiung.

Hsieh Chen, Ph.D. candidate, Institute of Science Education \& Environmental Education, National Kaohsiung Normal University. 
amygdala, anterior cingulate cortex, hippocampus, or insula participate. These areas of brain would affect our emotions and affections (Roxo, Franceschini, Zubaran, Kleber, \& Sander, 2011).

Although a lot of previous studies provide that the nature aromatherapy could decrease human's anxious and make them feel more relax, the concentration of nature aromatherapy did not be discussed more before. Does the human's anxious decrease more when the concentration of nature aromatherapy higher? Or the results would be conversed? The issue is very important and would be clarified in this study.

This study adopted electro encephalography (EEG), event related potentials (ERP), and questionnaire to be the instrument to know well the relationship between the concentration of nature aromatherapy and the human's anxious.

Van Dinteren, Arns, Jongsma and Kessels (2014) indicated that the brain frequency in EEG and ERP study can be defined as four frequencies. Theta frequency is $4-7 \mathrm{~Hz}$, Alpha frequency is $13-21 \mathrm{~Hz}$, low Beta frequency is $13-21 \mathrm{~Hz}$, and high Beta frequency is $22-30 \mathrm{~Hz}$. The analysis of neuroscience date in this research will follow the definition above.

\section{Research Design and Methodology}

In this study, three different aromatherapy would be produced. Bottle A is $25 \%$ concentration of nature lavender aromatherapy. Bottle B is $75 \%$ concentration of nature lavender aromatherapy. And Bottle C is pure air without any flavor to be the blank task. All of the bottles were glass with brown color and the bodies of bottles just show $\mathrm{A}, \mathrm{B}$, or $\mathrm{C}$ without any other words.

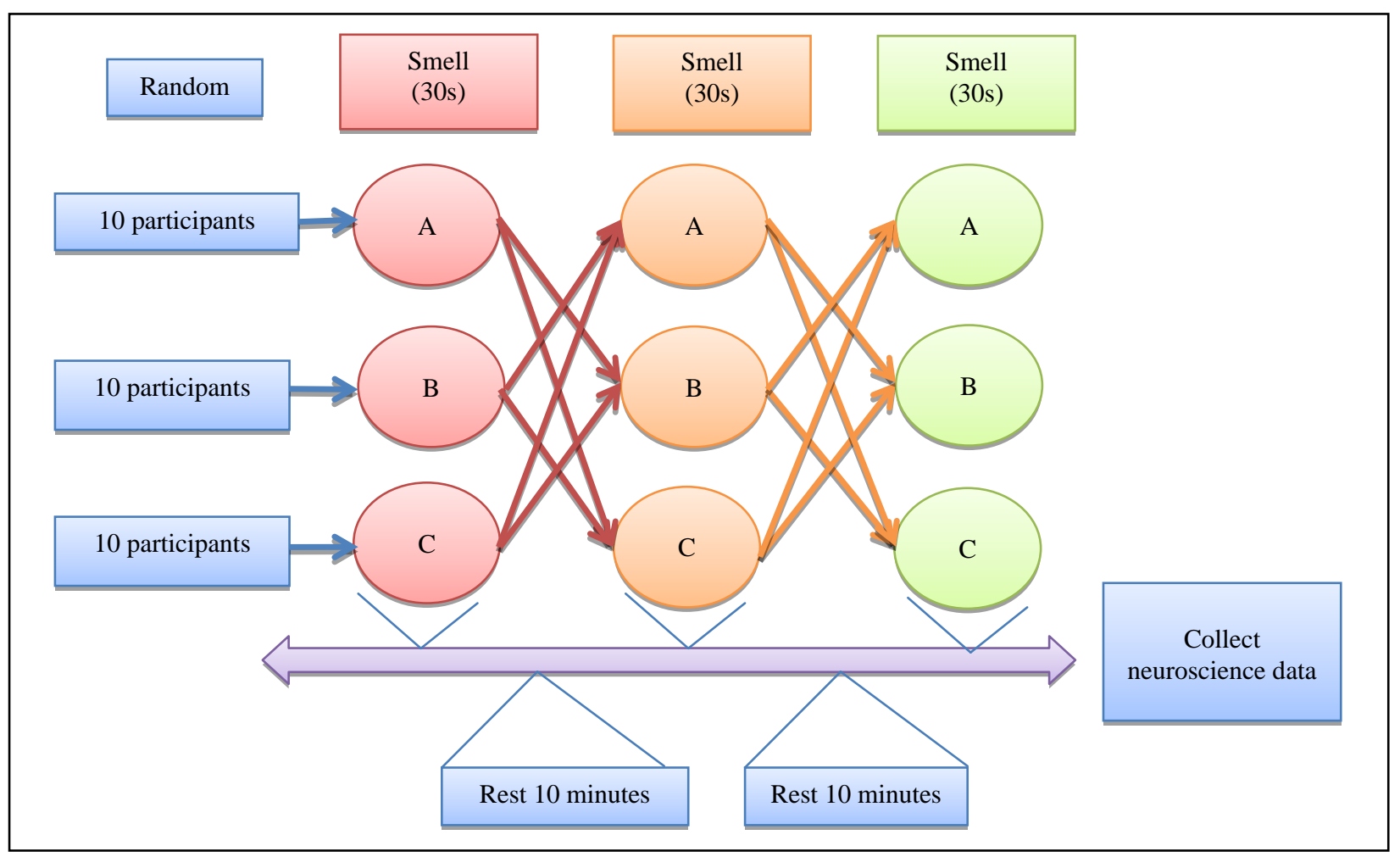

Figure 1. Research design.

There are 30 volunteers participated in this study. All of them would be divided into three groups randomly (see Figure 1). The first group would smell Bottle A first, and then smell B and C randomly; the 
second group would smell Bottle B first; and the third group would smell Bottle C first. The participants would smell each bottle for 30 seconds and take a rest for 10 minutes after smelling the next bottle. This study collected the participants' neuroscience data in the whole research.

\section{Participants}

There are 30 participants in this study (Male $=15$; Female $=15$; and Average age $=37$ years old). All of them are volunteers and signed in agreement consents before doing this research. All of them did not have any illness before and during this study.

\section{Instruments}

There are two instruments in this study. One is Beck Anxiety Inventory (BAI) and the other is neuroscience technology.

The BAI has been developed by Beck and Steer (1993). The reality reached in Cronbach's $\alpha=0.89$. There are 21 items included in this inventory, the participants would answer the inventory by self-report. Each answer being scored on a scale value of 0 ("Not at all”) to 3 ("Severely"). Higher total scores indicate more severe anxiety symptoms. The standardized cutoffs are: (1) 0-7: Minimal anxiety; (2) 8-15: Mild anxiety; (3) 16-25: Moderate anxiety; and (4) 26-63: Severe anxiety. In this study, the average scores would be analyzed. The average scores are as follows: (1) Normal: < 5 points; (2) Minimal anxiety: 5.1-10 points; (3) Moderate anxiety: 10.1-15 points; and (4) Severe anxiety: $>15.1$ points.

The other methodology is neuroscience technology. The technology included SynAmpTM amplifier, Stim, and Scan system. The amplifier is $1,000 \mathrm{~Hz}$, the filter is $0.1-200 \mathrm{~Hz}$, and the sample rate is $10,000 \mathrm{~Hz}$. The Scan system is SynAmps/Scan 4.4 (NeuroScan, Inc., Herndon, and VA). The brain wave cap included 36 electrode chanel (36 chanel, NeuroScan, USA) and the system is NeuroStim software.

\section{Results}

All participants were been analyzed their anxious situation recently. All participants were writing down their answers by self-report. Each answer being scored on a scale value of 0 ("Not at all”) to 3 ("Severely"). Higher total scores indicate more severe anxiety symptoms. In this study, the average scores would be analyzed. The average scores are as follows: (1) Normal: < 5 points; (2) Minimal anxiety: 5.1-10 points; (3) Moderate anxiety: 10.1-15 points; and (4) Severe anxiety: > 15.1 points.

The results from statistical analysis show that there were just $8 \%$ participants in this study felt normal without any anxious in their daily life (see Figure 2). There were 39\% participants in this study felt minimal anxiety, $41 \%$ participants felt moderate anxiety, and $12 \%$ participants felt several anxiety in this study.

The result from Figure 2 indicated that, we have totally 92\% participants in this study felt anxiety in their daily life. The physics and psychology disasters in the decade of a century might be from the highly anxiety.

For knowing well, if the nature lavender aromatherapy will be work for decreasing human being's anxious or not, the BAI has been used to detect the participants' anxious recently. Each participant need to smell Bottle $\mathrm{A}, \mathrm{B}$, and $\mathrm{C}$ randomly and write down the inventory. The results were analyzed by $t$-test and are shown in Table 1. 


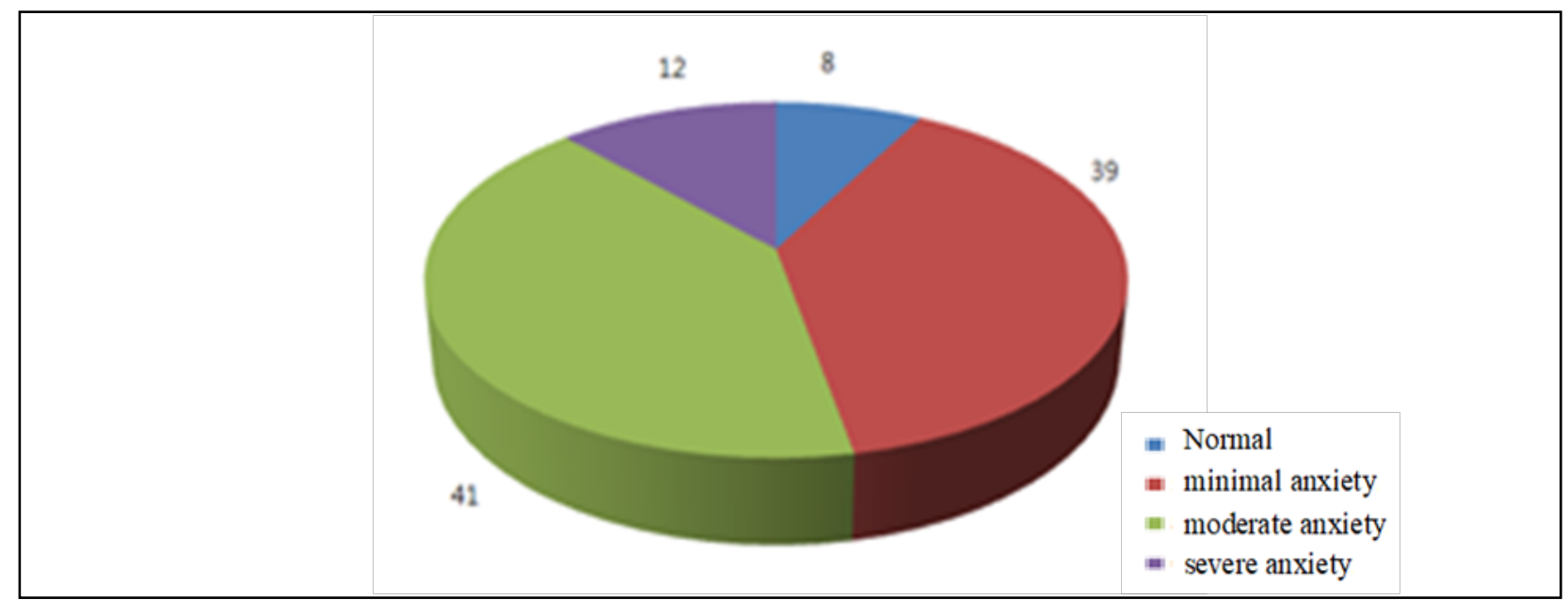

Figure 2. The participants' anxious situation in this study.

The results from Table 1 shows that the average scores of participants' anxious in this study is minimal anxiety but close to moderate anxiety. However, after they smelling the 25\% nature lavender aromatherapy, their average score of anxiety was decreased into $5.1 \pm 1.4$. The scores is minimal anxiety but very close to normal.

Then, we inferred that if the human being felt relax, their attention will be decreased. Van Dinteren et al. (2014) mentioned that in ERP studies, P300 component will be an important indicator for reflecting people's attention cognition, and the main reflection brain area are CZ and PZ electrode point (see Figure 3).

Table 1

The Different Performance of Anxious by Smelling Different Aromatherapy

\begin{tabular}{lll}
\hline Participants & Concentration of lavender aromatherapy & Avg. \pm SD \\
\hline \multirow{3}{*}{30 volunteers } & Bottle A (pure air) & $9.2 \pm 2.2$ \\
& Bottle B (25\% lavender aromatherapy) & $5.1 \pm 1.4$ \\
& Bottle C (75\% lavender aromatherapy) & $6.8 \pm 1.5$ \\
\hline
\end{tabular}

Notes. (1) Normal: < 5 points; (2) Minimal anxiety: 5.1-10 points; (3) Moderate anxiety: 10.1-15 points; and (4) Severe anxiety: > 15.1 points.

In ERP data, the P300 component in neuroscience raw data will be shown in Figure 4. In this study, we track the P300 components in neuroscience raw data and translate it into statistical data, and analyzed all the statistical data by using statistic package for social science (SPSS).

The results of the statistical data from neuroscience data are shown in Table 2. All the data have been analyzed by paired $t$-test.

The data in Table 2 confirmed the hypothesis. The participants will feel more relax after smelling lower concentration aromatherapy (Bottle B, 25\% lavender aromatherapy), and that will also decrease their attention. Oppositely, the higher concentration of lavender aromatherapy (Bottle C, 75\% lavender aromatherapy) could not decrease the participants' anxious, but it could increase human being's attention. These findings indicated that the different concentration of nature lavender aromatherapy had different functions for human beings. The users could apply all of them for different demands. 


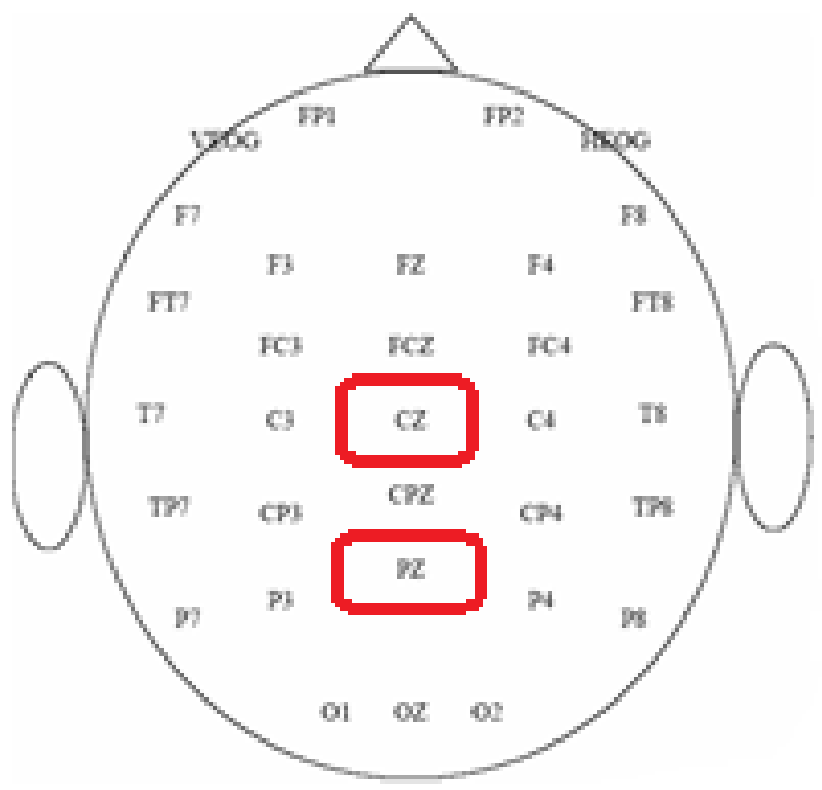

Figure 3. CZ and PZ electrode point in brain area.

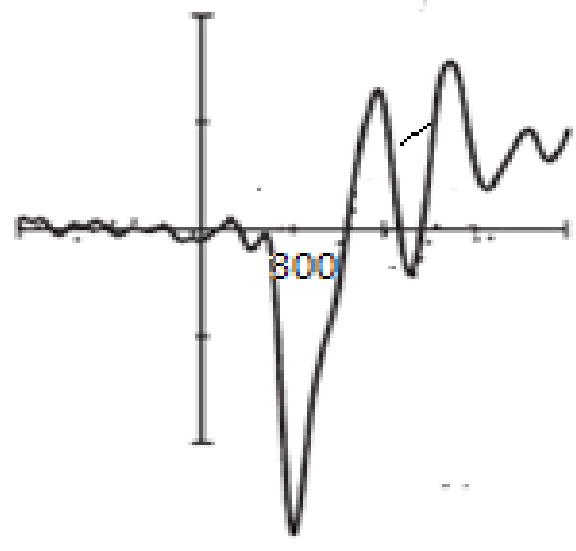

P300

Figure 4. P300 component in ERP raw data.

Table 2

Paired T-test of P300 Component

\begin{tabular}{llll}
\hline Factor & Pair & Avg. \pm SD & $t$ \\
\hline & Bottle B & $-2.7 \pm 1.9$ & $-.1 .83^{*}$ \\
P300 & Bottle A & $-0.8 \pm 2.4$ & $-5.76^{* * *}$ \\
(Amplitude value) & Bottle C & $-6.6 \pm 4.9$ & $-3.82^{* *}$ \\
& Bottle A & $-0.8 \pm 2.4$ & $-6.6 \pm 4.9$ \\
& Bottle C & $-2.7 \pm 1.9$ & \\
& Bottle B & -9.9 & \\
\hline
\end{tabular}

Notes. Bottle A is pure air; Bottle B is 25\% lavender aromatherapy; and Bottle C is $75 \%$ lavender aromatherapy; ${ }^{*} p<0.05 ;{ }^{* *} p<0.01$; and ${ }^{* * *} p<0.001$. 


\section{Conclusion}

This study aims to explore the aromatherapy effect and performance by using questionnaire and neuroscience technology (EEG and ERP). There are different concentration of nature lavender aromatherapy (25\% and $75 \%$ ) in this study and 30 participants will perform their attention and anxious after smelling these different concentration of aromatherapy. All of the participants need to write down the questionnaires about anxious, and would smell with carrying the brain wave cap to detect their neuroscience data. The results show that higher concentration of aromatherapy could induce the participants' attention, but lower concentration of aromatherapy could decrease the participants' anxious. The suggestions are that the different concentration of nature lavender aromatherapy had different functions for human beings. The users could apply all of them for different demands. Also, in this study, the lavender aromatherapy is nature. It was been extracted by water vapor without using any chemical solvents. The further researches might be pay attention for choosing the products of aromatherapy.

\section{References}

Beck, A. T., \& Steer, R. A. (1993). Beck anxiety inventory manual. San Antonio, TX: The Psychological Corporation Harcourt Brace \& Company.

Herz, R. S. (2009). Analysis of olfactory effects on mood, physiology and behavior. International Journal of Neuroscienc, 119(2), 263-290.

Liu, Y. Y., Yang, H. L., Chen, S. W., \& Wang, H. C. (2017). A nursing experience of a terminal cervical cancer patient. Tzu Chi Nursing Journal, 16(6), 94-105.

Nguyen, Q., \& Paton, C. (2008). The use of aromatherapy to treat behavioral problems in dementia. Journal of Geriatric Psychiatry, 23(4), 337-346.

Pekrun, R., Goetz, T., Titz, W., \& Perry, R. P. (2002). Positive emotions in education. Oxford, U.S.: Oxford University Press.

Van Dinteren, R., Arns, M., Jongsma, M. L. A., \& Kessels, R. P. C. (2014). P300 development across the lifespan: A systematic review and meta-analysis_PLOS one. Retrieve December 10, 2018, from https://journals.plos.org/plosone/article?id=10. 1371/journal.pone.0087347

Roxo, M. R., Franceschini, P. R., Zubaran, C., Kleber, F. D., \& Sander, J. W. (2011). The limbic system conception and its historical evolution. The Scientific World Journal, 11, 2427-2440. 